\title{
A Crank-Nicolson finite spectral element method for the 2D non-stationary Stokes equations about vorticity-stream functions
}

\section{Yanjie Zhou' ${ }^{1}$ Zhendong Luo ${ }^{2^{*}}$ (D) and Fei Teng ${ }^{2}$}

*Correspondence:

zhdluo@ncepu.edu.cn

${ }^{2}$ School of Mathematics and

Physics, North China Electric Power University, Beijing, China

Full list of author information is

available at the end of the article

\section{Springer}

\begin{abstract}
In this article, we first develop a semi-discretized Crank-Nicolson format about time for the two-dimensional non-stationary Stokes equations about vorticity-stream functions and analyze the existence, uniqueness, stability, and convergence of the semi-discretized Crank-Nicolson solutions. Then we establish a fully discretized Crank-Nicolson finite spectral element format based on the quadrilateral elements for the two-dimensional non-stationary Stokes equations about vorticity-stream functions and analyze the existence, uniqueness, stability, and convergence of the Crank-Nicolson finite spectral element solutions. In the end, we use three numerical examples to confirm the validity of our theoretical conclusions.
\end{abstract}

MSC: $65 \mathrm{~N} 30 ; 65 \mathrm{~N} 12 ; 65 \mathrm{M} 15$

Keywords: Semi-discretized Crank-Nicolson format; The two-dimensional non-stationary Stokes equations about vorticity-stream functions; Fully discretized Crank-Nicolson finite spectral element format; Existence and stability as well as convergence

\section{Introduction}

Let $\Theta \subset \mathbb{R}^{2}$ be a connected and bounded domain. Consider the following two-dimensional (2D) non-stationary Stokes equations:

Problem 1 Find $(u, v)$ and $p$ such that

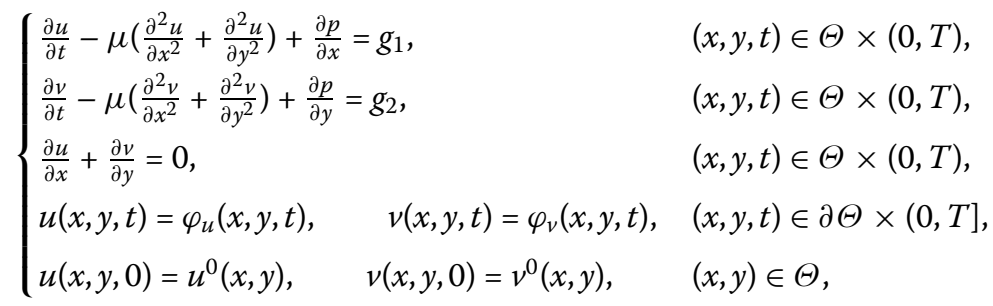

where $(u, v)$ represents the fluid velocity vector, $p$ is the pressure, $T$ is the total time, $\mu=$ $1 / R e, R e$ is the Reynolds number, $g_{1}(x, y, t), \varphi_{u}(x, y, t)$, and $u^{0}(x, y)$ are, respectively, the given body force, boundary value, and initial value functions in the $x$ direction, and $g_{2}(x, y, t)$,

(c) The Author(s) 2018. This article is distributed under the terms of the Creative Commons Attribution 4.0 International License (http://creativecommons.org/licenses/by/4.0/), which permits unrestricted use, distribution, and reproduction in any medium, provided you give appropriate credit to the original author(s) and the source, provide a link to the Creative Commons license, and indicate if changes were made. 
$\varphi_{v}(x, y, t), v^{0}(x, y)$ are, respectively, the given body force, boundary value, and initial value functions in the $y$ direction.

For the sake of convenience but without losing generality, we will assume that $\varphi_{u}(x, y, t)=$ $\varphi_{\nu}(x, y, t)=0$ in the following discussion.

The 2D non-stationary Stokes equations constitute an important mathematical model in fluid dynamics and have been successfully and extensively used to simulate the practical engineering problems as mentioned in [1-8]. However, when their computational domains are the irregular geometrical shape, we can usually not find their analytical solutions, so that we have to depend upon numerical solutions.

At present, finite difference (FD) scheme (see, e.g., $[9,10]$ ), finite element (FE) method (see, e.g., $[1-4,11,12])$, finite volume element (FVE) method (see, e.g., [13, 14]), and spectral method (see [15-21]) are considered as to be four popular numerical methods. However, the spectral method holds highest accuracy among four numerical methods because it adopts the whole smooth functions (such as trigonometric functions, Chebyshev's polynomials, Jacobi's polynomials, and Legendre's polynomials) to approximate unknown function, whereas the FE and FVE methods usually adopt standard polynomials to approximate unknown function and the FD scheme adopts difference quotient to approximate derivative. Especially, the finite spectral element (FSE) method can be suitable for the computational domains with complex geometric shapes, just as the FE method, so that it is widely used to solve various partial differential equations (PDEs), including the second-order elliptic equations, the parabolic equations, the hyperbolic equations, the hydromechanics equations (see, e.g., [22-26]).

Though some FSE methods have been presented in $[25,26]$, as far as we know, there has not been any report that the Crank-Nicolson (CN) finite spectral element (CNFSE) method is used to solve the 2D non-stationary Stokes equations about vorticity-stream functions, especially, there has not been any report about the theoretical analysis of the existence, stability, and convergence of the CNFSE solutions. Therefore, in this paper, we will first propose a time semi-discretized $\mathrm{CN}$ format with second-order time accuracy for the 2D non-stationary Stokes equations about vorticity-stream functions and analyze the errors of the time semi-discretized $\mathrm{CN}$ solutions. Then we will establish the fully discretized CNFSE format based on the quadrilateral elements for the 2D non-stationary Stokes equations about vorticity-stream functions and analyze the existence, uniqueness, stability, and convergence of the CNFSE solutions. In the end, we will use three numerical examples to confirm the validity of the obtained theoretical conclusions.

The CNFSE format for the 2D non-stationary Stokes equations about vorticity-stream functions has not only the second-order accuracy in time, but also is formed by system of two relatively independent linear equations for vorticity-stream approximate functions, so that it can easily be solved, which is different from the existing other FSE methods as mentioned above. Of course, the CNFSE format is also different from the spectral methods in [15-21]. Therefore, the CNFSE method here is a development and improvement over the existing methods.

The rest contents of this article is arranged as follows. In Sect. 2, we propose the semidiscretized $\mathrm{CN}$ format with approximation of second order by the time variable for the 2D non-stationary Stokes equations about vorticity-stream functions and analyze the existence, uniqueness, stability, and convergence of the time semi-discretized $\mathrm{CN}$ solutions. 
In Sect. 3, we establish the fully discretized CNFSE format based on the quadrilateral elements for the 2D non-stationary Stokes equations about vorticity-stream functions and analyze the existence, uniqueness, stability, and convergence of the CNFSE solutions. In Sect. 4, we use three numerical examples to confirm the validity of theoretical conclusion. Section 5 provides the main conclusions and discussions.

\section{The semi-discretized CN method about time for the 2D non-stationary Stokes equations}

The Sobolev spaces, norms, and inner products used in this article are common (see [27]).

\subsection{The semi-discretized CN format about time}

When $\Theta$ is connected and bounded and $\partial u / \partial x+\partial v / \partial y=0$, there is a unique stream function $\psi$ such that

$$
u=\frac{\partial \psi}{\partial y}, \quad v=-\frac{\partial \psi}{\partial x} .
$$

Further, there is unique a vorticity function $\omega$ such that $\omega=\partial v / \partial x-\partial u / \partial y=-\left(\partial \psi^{2} / \partial x^{2}+\right.$ $\left.\partial \psi^{2} / \partial y^{2}\right)$. Thus, Problem 1 can be transformed into the following system of two relatively independent linear PDEs about vorticity-stream functions.

Problem 2 Find $\omega$ and $\psi$ such that

$$
\begin{aligned}
& \begin{cases}-\frac{\partial \psi^{2}}{\partial x^{2}}-\frac{\partial \psi^{2}}{\partial y^{2}}=\omega, & (x, y, t) \in \Theta \times(0, T), \\
\psi=0, & (x, y, t) \in \partial \Theta \times[0, T],\end{cases} \\
& \begin{cases}\frac{\partial \omega}{\partial t}-\mu\left(\frac{\partial^{2} \omega}{\partial x^{2}}+\frac{\partial^{2} \omega}{\partial y^{2}}\right)=f, & (x, y, t) \in \Theta \times(0, T), \\
\omega=0, & (x, y, t) \in \partial \Theta \times[0, T], \\
\omega=\omega^{0}, & (x, y) \in \Theta,\end{cases}
\end{aligned}
$$

where $f=\partial g_{2} / \partial x-\partial g_{1} / \partial y, \omega^{0}=\partial \nu^{0} / \partial x-\partial u^{0} / \partial y$.

When $\left(g_{1}, g_{2}\right) \in H^{1}\left(0, T ; C^{1}(\bar{\Theta})\right) \times H^{1}\left(0, T ; C^{1}(\bar{\Theta})\right)$ and $\left(u^{0}, v^{0}\right) \in H^{2}(\Theta) \times H^{2}(\Theta)$, from the above discussion and the regularity for PDEs (see, e.g., $[11,27])$ we can conclude that there is a unique solution $\omega \in H^{2}\left(0, T ; C_{0}^{1}(\bar{\Theta}) \cap H^{2}(\Theta)\right)$ and $\psi \in H^{2}\left(0, T ; C_{0}^{1}(\bar{\Theta}) \cap H^{2}(\Theta)\right)$ for Problem 2 meeting

$$
\|\psi\|_{H^{2}\left(W^{0, \infty}\right)}+\|\psi\|_{H^{2}\left(H^{2}\right)}+\|\omega\|_{H^{2}\left(W^{0, \infty}\right)}+\|\omega\|_{H^{2}\left(H^{2}\right)} \leq \sigma\left(g_{1}, g_{2}, u^{0}, v^{0}, \mu\right)
$$

where $\|\cdot\|_{H^{m}\left(W^{0, \infty)}\right.}$ and $\|\cdot\|_{H^{m}\left(H^{k}\right)}$ represent, respectively, the norms in spaces $H^{m}(0, T$; $\left.W^{0, \infty}(\Theta)\right)$ and $H^{m}\left(0, T ; H^{k}(\Theta)\right)$, and $\sigma\left(g_{1}, g_{2}, u^{0}, v^{0}\right)$ is a non-negative constant dependent on $g_{1}, g_{2}, u^{0}, v^{0}$, and $\mu$.

Let $M$ be a positive integer, $\Delta t=T / M$ be the time step, and $\omega^{n}(x, y)$ and $\psi^{n}(x, y)$ be the approximations of $\omega(x, y, t)$ and $\psi(x, y, t)$ at $t_{n}=n \Delta t(n=0,1,2, \ldots, M)$, respectively. From the first equation in (4) we attain

$$
\frac{\partial^{2} \omega}{\partial t^{2}}=\frac{\partial}{\partial t}\left[\mu\left(\frac{\partial^{2} \omega}{\partial x^{2}}+\frac{\partial^{2} \omega}{\partial y^{2}}\right)+f\right]
$$


Thus, by Taylor's formula and (6) we obtain

$$
\begin{aligned}
\frac{\partial \omega^{n-1}}{\partial t} & =\frac{\omega^{n}-\omega^{n-1}}{\Delta t}-\frac{\Delta t}{2} \frac{\partial^{2} \omega^{n-1}}{\partial t^{2}}+o\left(\Delta t^{2}\right) \\
& =\frac{\omega^{n}-\omega^{n-1}}{\Delta t}-\frac{\mu}{2}\left(\frac{\partial^{2}\left(\omega^{n}-\omega^{n-1}\right)}{\partial x^{2}}+\frac{\partial^{2}\left(\omega^{n}-\omega^{n-1}\right)}{\partial y^{2}}\right)+\frac{f^{n}-f^{n-1}}{2}+o\left(\Delta t^{2}\right) .
\end{aligned}
$$

Further, by inputting (7) into the first equation in (4) we obtain

$$
\frac{\omega^{n}-\omega^{n-1}}{\Delta t}-\frac{\mu}{2}\left[\frac{\partial^{2}\left(\omega^{n}+\omega^{n-1}\right)}{\partial x^{2}}+\frac{\partial^{2}\left(\omega^{n}+\omega^{n-1}\right)}{\partial y^{2}}\right]=\frac{f^{n}+f^{n-1}}{2}+o\left(\Delta t^{2}\right)
$$

Set $V=H_{0}^{1}(\Theta)$. Thus, by Green's formula we can establish the semi-discretized CN format with the second-order accuracy in time as follows.

Problem 3 For given $\omega^{0} \in C^{0}(\bar{\Theta})$ and $f^{n} \in C^{0}(\bar{\Theta})(n=0,1, \ldots, M)$, find $\left(\omega^{n}, \psi^{n}\right) \in V \times V$ $(n=1,2, \ldots, M)$ that satisfy

$$
\begin{aligned}
& \int_{\Theta}\left(\frac{\partial \psi^{n-1}}{\partial x} \frac{\partial w}{\partial x}+\frac{\partial \psi^{n-1}}{\partial y} \frac{\partial w}{\partial y}\right) \mathrm{d} x \mathrm{~d} y \\
& =\int_{\Theta} \omega^{n-1} w \mathrm{~d} x \mathrm{~d} y, \quad \forall w \in V, n=1,2, \ldots, M+1 \\
& \int_{\Theta}\left[\omega^{n} w+\frac{\mu \Delta t}{2}\left(\frac{\partial \omega^{n}}{\partial x} \frac{\partial w}{\partial x}+\frac{\partial \omega^{n}}{\partial y} \frac{\partial w}{\partial y}\right)\right] \mathrm{d} x \mathrm{~d} y \\
& =\int_{\Theta}\left[\omega^{n-1} w-\frac{\mu \Delta t}{2}\left(\frac{\partial \omega^{n-1}}{\partial x} \frac{\partial w}{\partial x}+\frac{\partial \omega^{n-1}}{\partial y} \frac{\partial w}{\partial y}\right)\right] \mathrm{d} x \mathrm{~d} y \\
& \quad+\frac{\Delta t}{2} \int_{\Theta}\left(f^{n}+f^{n-1}\right) w \mathrm{~d} x \mathrm{~d} y, \quad \forall w \in V, n=1,2, \ldots, M .
\end{aligned}
$$

\subsection{The existence, uniqueness, stability, and convergence of the time semi-discretized $\mathrm{CN}$ solutions}

In the following, we employ the Lax-Milgram theorem, and the Hölder, Poincaré, Cauchy-Schwarz inequalities, and the following discrete Gronwall inequality (see [11, Lemma 3.4] or [28, Lemma 1.4.1]) to analyze the existence, uniqueness, stability, and convergence for the time semi-discretized $\mathrm{CN}$ solutions to Problem 3.

Lemma 4 If $\left\{a_{n}\right\}$ and $\left\{b_{n}\right\}$ are two non-negative sequences, and $\left\{c_{n}\right\}$ is a positive monotone sequence, that satisfy

$$
a_{n}+b_{n} \leq c_{n}+\bar{\lambda} \sum_{i=0}^{n-1} a_{i} \quad(\bar{\lambda}>0) ; \quad a_{0}+b_{0} \leq c_{0}
$$

then

$$
a_{n}+b_{n} \leq c_{n} \exp (n \bar{\lambda}), \quad n=0,1,2, \ldots
$$

We have the following main conclusion for Problem 3. 
Theorem 5 If $\omega^{0} \in C^{0}(\bar{\Theta})$ and $f^{n} \in C^{0}(\bar{\Theta})$, i.e., $\left(u^{0}, v^{0}\right) \in C^{1}(\bar{\Theta}) \times C^{1}(\bar{\Theta})$ and $\left(g_{1}^{n}, g_{2}^{n}\right) \in$ $C^{1}(\bar{\Theta}) \times C^{1}(\bar{\Theta})$, the iterative equations (9) and (10) have a unique series of solutions $\left(\omega^{n}, \psi^{n}\right) \in V \times V(n=1,2, \ldots, M)$ meeting the following stability:

$$
\begin{aligned}
& \left\|\omega^{n}\right\|_{0}^{2}+\mu \Delta t\left\|\nabla \omega^{n}\right\|_{0}^{2} \leq\left(2\left\|\omega^{0}\right\|_{0}^{2}+\mu \Delta t\left\|\nabla \omega^{0}\right\|_{0}^{2}+2 \Delta t \sum_{i=0}^{n}\left\|f^{i}\right\|_{0}^{2}\right) \exp (2 n \Delta t), \\
& n=1,2, \ldots, M \\
& \left\|\nabla \psi^{n}(x, y)\right\|_{0} \leq \sigma\left(2\left\|\omega^{0}\right\|_{0}^{2}+\mu \Delta t\left\|\nabla \omega^{0}\right\|_{0}^{2}+2 \Delta t \sum_{i=0}^{n}\left\|f^{i}\right\|_{0}^{2}\right)^{1 / 2} \exp (2 n \Delta t), \\
& n=0,1,2, \ldots, M,
\end{aligned}
$$

and the following convergence:

$$
\left\|\psi\left(x, y, t_{n}\right)-\psi^{n}(x, y)\right\|_{0, \infty}+\left\|\omega\left(x, y, t_{n}\right)-\omega^{n}(x, y)\right\|_{0, \infty} \leq \sigma \Delta t^{2}
$$

where $\sigma$, used in the subsequent, is the generic positive constant independent of $\Delta t$, but it is inequable in different places.

\section{Proof Set}

$$
\begin{aligned}
& \tilde{A}(\psi, w)=\int_{\Theta}\left(\frac{\partial \psi}{\partial x} \frac{\partial w}{\partial x}+\frac{\partial \psi}{\partial y} \frac{\partial w}{\partial y}\right) \mathrm{d} x \mathrm{~d} y, \quad \forall \psi, w \in V ; \\
& \tilde{G}(w)=\int_{\Theta} \omega^{n-1} w \mathrm{~d} x \mathrm{~d} y, \quad \forall w \in V ; \\
& \tilde{B}(\omega, w)=\int_{\Theta}\left[\omega w+\frac{\mu \Delta t}{2}\left(\frac{\partial \omega}{\partial x} \frac{\partial w}{\partial x}+\frac{\partial \omega}{\partial y} \frac{\partial w}{\partial y}\right)\right] \mathrm{d} x \mathrm{~d} y, \quad \forall \omega, w \in V ; \\
& \tilde{F}(w)=\int_{\Theta}\left[\omega^{n-1} w-\frac{\mu \Delta t}{2}\left(\frac{\partial \omega^{n-1}}{\partial x} \frac{\partial w}{\partial x}+\frac{\partial \omega^{n-1}}{\partial y} \frac{\partial w}{\partial y}\right)\right] \mathrm{d} x \mathrm{~d} y \\
& +\frac{\Delta t}{2} \int_{\Theta}\left(f^{n}+f^{n-1}\right) w \mathrm{~d} x \mathrm{~d} y, \quad \forall w \in V .
\end{aligned}
$$

Then Problem 3 can be rewritten as follows.

Problem 6 Find $\left(\omega^{n}, \psi^{n}\right) \in V \times V(n=1,2, \ldots, M)$ that satisfy

$$
\begin{aligned}
& \tilde{A}\left(\psi^{n-1}, w\right)=\tilde{G}(w), \quad \forall w \in V, n=1,2, \ldots, M+1 ; \\
& \tilde{B}\left(\omega^{n}, w\right)=\tilde{F}(w), \quad \forall w \in V, n=1,2, \ldots, M .
\end{aligned}
$$

Both bilinear functionals $\tilde{A}(\cdot, \cdot)$ and $\tilde{B}(\cdot, \cdot)$ are bounded and positive definitive on $V \times V$ and both linear functionals $\tilde{G}(\cdot)$ and $\tilde{F}(\cdot)$ are bounded on $V$ for any given $\omega^{n-1}, f^{n}$, and $f^{n-1}$. Then, according on the Lax-Milgram theorem, Problem 6, i.e., the iterative equations (9) and (10), have a unique series of solutions $\left(\omega^{n}, \psi^{n}\right) \in V \times V(n=1,2, \ldots, M)$.

By taking $w=\psi^{n-1}$ in (9) in addition to the Hölder and Poincaré inequalities we get

$$
\left\|\nabla \psi^{n-1}\right\|_{0}^{2} \leq\left\|\omega^{n-1}\right\|_{0}\left\|\psi^{n-1}\right\|_{0} \leq \sigma\left\|\omega^{n-1}\right\|_{0}\left\|\nabla \psi^{n-1}\right\|_{0}, \quad n=1,2, \ldots, M+1 .
$$


Further, we obtain

$$
\left\|\nabla \psi^{n-1}\right\|_{0} \leq \sigma\left\|\omega^{n-1}\right\|_{0}, \quad n=1,2, \ldots, M+1
$$

By taking $w=\omega^{n}$ in (10) and the Hölder and Cauchy-Schwarz inequalities we obtain

$$
\begin{aligned}
\left\|\omega^{n}\right\|_{0}^{2}+\frac{\mu \Delta t}{2}\left\|\nabla \omega^{n}\right\|_{0}^{2} \\
\leq\left\|\omega^{n-1}\right\|_{0}\left\|\omega^{n}\right\|_{0}+\frac{\mu \Delta t}{2}\left\|\nabla \omega^{n-1}\right\|_{0}\left\|\nabla \omega^{n}\right\|_{0} \\
\quad+\frac{\Delta t}{2}\left\|\omega^{n}\right\|_{0}\left\|f^{n}\right\|_{0}+\frac{\Delta t}{2}\left\|\omega^{n}\right\|_{0}\left\|f^{n-1}\right\|_{0} \\
\leq \frac{1}{2}\left\|\omega^{n-1}\right\|_{0}^{2}+\frac{1}{2}\left\|\omega^{n}\right\|_{0}^{2}+\frac{\mu \Delta t}{4}\left\|\nabla \omega^{n-1}\right\|_{0}^{2}+\frac{\mu \Delta t}{4}\left\|\nabla \omega^{n}\right\|_{0} \\
\quad+\frac{\Delta t}{2}\left\|\omega^{n}\right\|_{0}^{2}+\frac{\Delta t}{4}\left(\left\|f^{n}\right\|_{0}^{2}+\left\|f^{n-1}\right\|_{0}^{2}\right), \quad n=1,2, \ldots, M .
\end{aligned}
$$

Further, we obtain

$$
\begin{aligned}
& \left\|\omega^{n}\right\|_{0}^{2}+\frac{\mu \Delta t}{2}\left\|\nabla \omega^{n}\right\|_{0}^{2} \\
& \leq\left\|\omega^{n-1}\right\|_{0}^{2}+\frac{\mu \Delta t}{2}\left\|\nabla \omega^{n-1}\right\|_{0}^{2} \\
& \quad+\Delta t\left\|\omega^{n}\right\|_{0}^{2}+\frac{\Delta t}{2}\left(\left\|f^{n}\right\|_{0}^{2}+\left\|f^{n-1}\right\|_{0}^{2}\right), \quad n=1,2, \ldots, M .
\end{aligned}
$$

Summing (23) from 1 to $n$ yields

$$
\begin{aligned}
& \left\|\omega^{n}\right\|_{0}^{2}+\frac{\mu \Delta t}{2}\left\|\nabla \omega^{n}\right\|_{0}^{2} \\
& \quad \leq\left\|\omega^{0}\right\|_{0}^{2}+\frac{\mu \Delta t}{2}\left\|\nabla \omega^{0}\right\|_{0}^{2}+\Delta t \sum_{i=1}^{n}\left\|\omega^{i}\right\|_{0}^{2}+\Delta t \sum_{i=0}^{n}\left\|f^{i}\right\|_{0}^{2}, \quad n=1,2, \ldots, M .
\end{aligned}
$$

When $\Delta t$ is sufficiently small such that $\Delta t \leq 1 / 2$, from (24), we attain

$$
\begin{aligned}
& \left\|\omega^{n}\right\|_{0}^{2}+\mu \Delta t\left\|\nabla \omega^{n}\right\|_{0}^{2} \\
& \quad \leq 2\left\|\omega^{0}\right\|_{0}^{2}+\mu \Delta t\left\|\nabla \omega^{0}\right\|_{0}^{2}+2 \Delta t \sum_{i=0}^{n-1}\left\|\omega^{i}\right\|_{0}^{2}+2 \Delta t \sum_{i=0}^{n}\left\|f^{i}\right\|_{0}^{2}, \quad n=1,2, \ldots, M .
\end{aligned}
$$

By using the discrete Gronwall inequality (Lemma 4) for (25), we obtain

$$
\begin{aligned}
& \left\|\omega^{n}\right\|_{0}^{2}+\mu \Delta t\left\|\nabla \omega^{n}\right\|_{0}^{2} \leq\left(2\left\|\omega^{0}\right\|_{0}^{2}+\mu \Delta t\left\|\nabla \omega^{0}\right\|_{0}^{2}+2 \Delta t \sum_{i=0}^{n}\left\|f^{i}\right\|_{0}^{2}\right) \exp (2 n \Delta t), \\
& n=1,2, \ldots, M
\end{aligned}
$$

This is exactly (11). By (11) and (21) we immediately attain (12).

By (8) we immediately attain (13). 


\section{The CNFSE method for the 2D non-stationary Stokes equations about vorticity-stream functions}

\subsection{The establishment of the CNFSE format}

Let $\Im_{N}$ be the quasi-uniform quadrilateral subdivision on $\bar{\Theta}$ and the spectral element subspace be chosen as the following:

$$
V_{N}=\left\{w_{N} \in H_{0}^{1}(\Theta) \cap C^{0}(\bar{\Theta}):\left.w_{N}\right|_{K_{j}} \in \mathcal{P}_{1}\left(K_{j}\right), K_{j} \in \Im_{N}, j=1,2, \ldots, N\right\}
$$

where $N$ is the number of elements and $\mathcal{P}_{1}\left(K_{j}\right)$ is formed by the quadrilateral spectral element, i.e.,

$$
\mathcal{P}_{1}\left(K_{j}\right)=\operatorname{span}\left\{N_{i j}: 1 \leq i \leq 4\right\},
$$

the above $N_{i j}=\hat{N}_{i} \circ F_{j}^{-1}(x, y), \hat{N}_{i}(\xi, \eta)=\left[1+\cos \pi\left(\xi-\xi_{i}\right)\right]\left[1+\cos \pi\left(\eta-\eta_{i}\right)\right] / 4,(x, y)=$ $F_{j}(\xi, \eta)=\left(\sum_{i=1}^{4} \hat{N}_{i}(\xi, \eta) x_{i j}, \sum_{i=1}^{4} \hat{N}_{i}(\xi, \eta) y_{i j}\right)$ is a reversible transformation from $K_{j} \in \Im_{N}$ to the referencing quadrilateral $\hat{K}=[-1,1] \times[-1,1]$, and $\left(x_{i j}, y_{i j}\right)$ and $\left(\xi_{i}, \eta_{i}\right)$ are the vertices of $K_{j}$ and $\hat{K}$, respectively (see $[11,25]$ ).

Let $R_{N}: H_{0}^{1}(\Theta) \rightarrow V_{N}$ be the $H^{1}$-orthogonal projection, i.e., for any $\varphi \in H_{0}^{1}(\Theta)$,

$$
\int_{\Theta} \nabla\left(R_{N} \varphi-\varphi\right) \nabla v_{N} \mathrm{~d} x \mathrm{~d} y=0, \quad \forall v_{N} \in V_{N}
$$

Further, because $\mathfrak{I}_{N}$ is the quasi-uniform quadrilateral subdivision for $\Theta$, the number of nodes is approximately equal to the number of elements (see [11, Lemma 1.30]), $R_{N}$ has the following important property (see, e.g., [17, Chapters II and III]).

Theorem 7 For any $\varphi \in H^{q}(\Omega)$ with $q \geq 2$, we have

$$
\left\|\nabla R_{N} \varphi\right\|_{0, r} \leq \sigma_{r}\|\nabla \varphi\|_{0, r}, \quad\left\|\partial^{k}\left(R_{N} \varphi-\varphi\right)\right\|_{0} \leq \sigma N^{k-q}, \quad 0 \leq k \leq q \leq N+1,
$$

where $\sigma_{r}\left(r=2\right.$ or $\infty$, and when $\left.r=2, \sigma_{r}=1\right)$ is the general positive constant independent of $N$ and $N$ is the number of nodes in $\mathfrak{\Im}_{N}$.

By the subspace $V_{N}$ we can establish the CNFSE formulation as follows.

Problem 8 Find $\left(\omega_{N}^{n}, \psi_{N}^{n}\right) \in V_{N} \times V_{N}(n=1,2, \ldots, M)$ that satisfy

$$
\begin{aligned}
& \int_{\Theta}\left(\frac{\partial \psi_{N}^{n-1}}{\partial x} \frac{\partial w_{N}}{\partial x}+\frac{\partial \psi_{N}^{n-1}}{\partial y} \frac{\partial w_{N}}{\partial y}\right) \mathrm{d} x \mathrm{~d} y \\
& =\int_{\Theta} \omega_{N}^{n-1} w_{N} \mathrm{~d} x \mathrm{~d} y, \quad \forall w_{N} \in V_{N}, n=1,2, \ldots, M+1 \\
& \int_{\Theta}\left[\omega_{N}^{n} w_{N}+\frac{\mu \Delta t}{2}\left(\frac{\partial \omega_{N}^{n}}{\partial x} \frac{\partial w_{N}}{\partial x}+\frac{\partial \omega_{N}^{n}}{\partial y} \frac{\partial w_{N}}{\partial y}\right)\right] \mathrm{d} x \mathrm{~d} y \\
& =\frac{\Delta t}{2} \int_{\Theta}\left(f^{n}+f^{n-1}\right) w_{N} \mathrm{~d} x \mathrm{~d} y \\
& \quad+\int_{\Theta}\left[\omega_{N}^{n-1} w_{N}+\frac{\mu \Delta t}{2}\left(\frac{\partial \omega_{N}^{n-1}}{\partial x} \frac{\partial w_{N}}{\partial x}+\frac{\partial \omega_{N}^{n-1}}{\partial y} \frac{\partial w_{N}}{\partial y}\right)\right] \mathrm{d} x \mathrm{~d} y
\end{aligned}
$$




$$
\begin{aligned}
& -\mu \Delta t \int_{\Theta}\left(\frac{\partial \omega_{N}^{n-1}}{\partial x} \frac{\partial w_{N}}{\partial x}+\frac{\partial \omega_{N}^{n-1}}{\partial y} \frac{\partial w_{N}}{\partial y}\right) \mathrm{d} x \mathrm{~d} y, \\
& \forall w_{N} \in V_{N}, n=1,2, \ldots, M
\end{aligned}
$$

where $\omega_{N}^{0}=R_{N} \omega^{0}$.

Set

$$
\begin{aligned}
& \boldsymbol{\omega}^{n}=\left(\omega_{11}^{n}, \omega_{21}^{n}, \omega_{31}^{n}, \omega_{41}^{n}, \omega_{12}^{n}, \omega_{22}^{n}, \omega_{32}^{n}, \omega_{42}^{n}, \ldots, \omega_{1 N}^{n}, \omega_{2 N}^{n}, \omega_{3 N}^{n}, \omega_{4 N}^{n}\right)^{T}, \\
& \boldsymbol{\psi}^{n}=\left(\psi_{11}^{n}, \psi_{21}^{n}, \psi_{31}^{n}, \psi_{41}^{n}, \psi_{12}^{n}, \psi_{22}^{n}, \psi_{32}^{n}, \psi_{42}^{n}, \ldots, \psi_{1 N}^{n}, \psi_{2 N}^{n}, \psi_{3 N}^{n}, \psi_{4 N}^{n}\right)^{T}, \\
& \tilde{\boldsymbol{N}}=\left(N_{11}, N_{21}, N_{31}, N_{41}, N_{12}, N_{22}, N_{32}, N_{42}, \ldots, N_{1 N}, N_{2 N}, N_{3 N}, N_{4 N}\right)^{T}, \\
& \omega_{N}^{n}=\sum_{j=1}^{N} \sum_{i=1}^{4} \omega_{i j}^{n} N_{i j}=: \tilde{\boldsymbol{N}} \cdot \boldsymbol{\omega}^{n}, \quad \psi_{N}^{n}=\sum_{j=1}^{N} \sum_{i=1}^{4} \psi_{i j}^{n} N_{i j}=: \tilde{\boldsymbol{N}} \cdot \boldsymbol{\psi}^{n} .
\end{aligned}
$$

Thus, Problem 8 can be rewritten as follows.

Problem 9 Find $\left(\omega^{n}, \boldsymbol{\psi}^{n}\right) \in \mathbb{R}^{4 N} \times \mathbb{R}^{4 N}(n=1,2, \ldots, M)$ that satisfy

$$
\begin{aligned}
& \hat{\boldsymbol{A}} \boldsymbol{\psi}^{n-1}=\boldsymbol{C} \boldsymbol{\omega}^{n-1}, \quad n=1,2, \ldots, M+1 ; \\
& \boldsymbol{A} \boldsymbol{\omega}^{n}=\boldsymbol{A} \boldsymbol{\omega}^{n-1}+\Delta t \boldsymbol{B} \boldsymbol{\omega}^{n-1}+\Delta t \boldsymbol{F}^{n}, \quad n=1,2, \ldots, M,
\end{aligned}
$$

where $\hat{\boldsymbol{A}}=\operatorname{diag}\left\{\hat{\boldsymbol{A}}_{11}, \hat{\boldsymbol{A}}_{22}, \ldots, \hat{\boldsymbol{A}}_{N N}\right\}, \boldsymbol{A}=\operatorname{diag}\left\{\boldsymbol{A}_{11}, \boldsymbol{A}_{22}, \ldots, \boldsymbol{A}_{N N}\right\}, \boldsymbol{B}=\operatorname{diag}\left\{\boldsymbol{B}_{11}, \boldsymbol{B}_{22}, \ldots, \boldsymbol{B}_{N N}\right\}$, $\boldsymbol{C}=\operatorname{diag}\left\{\boldsymbol{C}_{11}, \boldsymbol{C}_{22}, \ldots, \boldsymbol{C}_{N N}\right\}, \boldsymbol{F}^{n}=\left(\boldsymbol{F}_{I \times 1}^{n}\right)_{4 N \times 1}$, and

$$
\begin{aligned}
\hat{\boldsymbol{A}}_{I J} & =\left(\int_{\Theta}\left[\frac{\partial N_{i I}}{\partial x} \frac{\partial N_{j J}}{\partial x}+\frac{\partial N_{i I}}{\partial y} \frac{\partial N_{j J}}{\partial y}\right] \mathrm{d} x \mathrm{~d} y\right)_{4 \times 4}, \\
\boldsymbol{A}_{I J} & =\left(\int_{\Theta}\left[N_{i I} N_{j J}+\frac{\mu \Delta t}{2}\left(\frac{\partial N_{i I}}{\partial x} \frac{\partial N_{j J}}{\partial x}+\frac{\partial N_{i I}}{\partial y} \frac{\partial N_{j J}}{\partial y}\right)\right] \mathrm{d} x \mathrm{~d} y\right)_{4 \times 4}, \\
\boldsymbol{B}_{I J} & =-\mu\left(\int_{\Theta}\left(\frac{\partial N_{i I}}{\partial x} \frac{\partial N_{j J}}{\partial x}+\frac{\partial N_{i I}}{\partial y} \frac{\partial N_{j J}}{\partial y}\right) \mathrm{d} x \mathrm{~d} y\right)_{4 \times 4}, \\
\boldsymbol{C}_{I J} & =\left(\int_{\Theta} N_{i I} N_{j J} \mathrm{~d} x \mathrm{~d} y\right)_{4 \times 4}, \quad \boldsymbol{F}_{I \times 1}^{n}=\left(\int_{\Theta} \frac{f^{(n)}+f^{(n-1)}}{2} N_{j I} \mathrm{~d} x \mathrm{~d} y\right)_{4 \times 1} .
\end{aligned}
$$

\subsection{The existence, stability, and convergence of the CNFSE solutions}

To analyze the existence, stability, and convergence of the CNFSE solutions, we consider the max-norms of matrix and vector (the more detailed results see [26]), which are, respectively, defined dy

$$
\begin{aligned}
& \|\boldsymbol{D}\|_{\infty}=\max _{1 \leq i \leq m} \sum_{j=1}^{l}\left|d_{i j}\right|, \quad \forall \boldsymbol{D}=\left(d_{i j}\right)_{m \times l} \in \mathbb{R}^{m} \times \mathbb{R}^{l}, \\
& \|\boldsymbol{\chi}\|_{\infty}=\max _{1 \leq j \leq m}\left|\chi_{j}\right|, \quad \forall \boldsymbol{\chi}=\left(\chi_{1}, \chi_{2}, \ldots, \chi_{m}\right)^{T} \in \mathbb{R}^{m} .
\end{aligned}
$$


In the following, we employ the matrix theory, the FE (see [11]) and FSE (see [17]) methods, and the discrete Gronwall (Lemma 4), Hölder, Poincaré, and Cauchy-Schwarz inequalities to analyze the existence, stability, and convergence of the CNFSE solutions for Problem 8 . We have the following result.

Theorem 10 If $\omega^{0}=\partial v^{0} / \partial x-\partial u^{0} / \partial y \in W^{0, \infty}(\Theta)$, i.e., $\left(u^{0}, v^{0}\right) \in W^{1, \infty}(\Theta) \times W^{1, \infty}(\Theta)$, and $f=\partial g_{2} / \partial x-\partial g_{1} / \partial y \in W^{0, \infty}(\Theta)$, i.e., $\left(g_{1}, g_{2}\right) \in W^{1, \infty}(\Theta) \times W^{1, \infty}(\Theta)$, then the CNFSE solutions $\left(\omega_{N}^{n}, \psi_{N}^{n}\right)$ are existing and unique and satisfy the following stability:

$$
\begin{aligned}
& \left\|\omega_{N}^{n}\right\|_{0, \infty} \leq \sigma\left(\left\|\omega^{0}\right\|_{\infty}+\Delta t N^{-1} \sum_{i=1}^{n}\left\|\boldsymbol{F}^{i}\right\|_{\infty}\right), \quad n=1,2, \ldots, M, \\
& \left\|\psi_{N}^{n}\right\|_{0, \infty} \leq \sigma N^{-1}\left(\left\|\omega^{0}\right\|_{\infty}+\Delta t N^{-1} \sum_{i=1}^{n}\left\|\boldsymbol{F}^{i}\right\|_{\infty}\right), \quad n=1,2, \ldots, M .
\end{aligned}
$$

where $\sigma$, used in the subsequent, is also the generic positive constant independent of $\Delta t$ and $N$, but is inequable in different places. Further, when $(\omega, \psi) \in\left[H^{3}\left(0, T ; H^{q}(\Theta) \cap H_{0}^{1}(\Theta)\right)\right] \times$ $\left[H^{3}\left(0, T ; H^{q}(\Theta) \cap H_{0}^{1}(\Theta)\right)\right](2 \leq q \leq N+1)$, we have the following error estimates:

$$
\begin{aligned}
& \left\|\omega\left(x, y, t_{n}\right)-\omega_{N}^{n}\right\|_{0}+\sqrt{\Delta t}\left\|\nabla\left(\omega\left(x, y, t_{n}\right)-\omega_{N}^{n}\right)\right\|_{0} \leq \sigma\left(\Delta t^{2}+N^{1-q}\right) ; \\
& \left\|\nabla\left(\psi\left(x, y, t_{n}\right)-\psi_{N}^{n}\right)\right\|_{0} \leq \sigma\left(\Delta t^{2}+N^{1-q}\right),
\end{aligned}
$$

where $n=1,2, \ldots, M$.

Proof First, by the symmetry and positive definiteness of the matrices $\hat{\boldsymbol{A}}$ and $\boldsymbol{A}$ we conclude that Problem 9 has a unique series of the coefficient vector solutions $\left(\omega^{n}, \boldsymbol{\psi}^{n}\right) \in \mathbb{R}^{4 N} \times \mathbb{R}^{4 N}$ $(n=1,2, \ldots, M)$. Thus, by $\omega_{N}^{n}=\tilde{\boldsymbol{N}} \cdot \boldsymbol{\omega}^{n}, \psi_{N}^{n}=\tilde{\boldsymbol{N}} \cdot \boldsymbol{\psi}^{n}$ we can immediately conclude that Problem 8 has a unique series of the CNFSE solutions $\left(\omega_{N}^{n}, \psi_{N}^{n}\right)(n=1,2, \ldots, M)$.

Next, we analyze the stability of the CNFSE solutions. From (31) and (32) we can attain the following:

$$
\begin{cases}\boldsymbol{\psi}^{n-1}=\hat{\boldsymbol{A}}^{-1} \boldsymbol{C} \boldsymbol{\omega}^{n-1}, & 1 \leq n \leq M+1 \\ \boldsymbol{\omega}^{n}=\boldsymbol{\omega}^{n-1}+\Delta t \boldsymbol{A}^{-1} \boldsymbol{B} \boldsymbol{\omega}^{n-1}+\Delta t \boldsymbol{A}^{-1} \boldsymbol{F}^{n}, & 1 \leq n \leq M\end{cases}
$$

Moreover, from the FE method (see, e.g., [11, Lemmas 1.18 and 1.22]) and FSE method (see, e.g., [17, Chapters II and III]) we can attain the following inequalities:

$$
\begin{aligned}
& \left\|\hat{\boldsymbol{A}}^{-1}\right\|_{\infty} \leq \sigma N^{-1} ; \quad\left\|\boldsymbol{A}^{-1}\right\|_{\infty} \leq \sigma N^{-1} ; \quad\|\boldsymbol{B}\|_{\infty} \leq \sigma N \\
& \|\boldsymbol{C}\|_{\infty} \leq \sigma, \quad\left\|\boldsymbol{C}^{-1}\right\|_{\infty} \leq \sigma
\end{aligned}
$$

Thus, by (37) and (38) we obtain

$$
\begin{aligned}
& \left\|\boldsymbol{\psi}^{n}\right\|_{\infty} \leq \sigma N^{-1}\left\|\boldsymbol{\omega}^{n}\right\|_{\infty}, \quad n=0,1,2, \ldots, M \\
& \left\|\boldsymbol{\omega}^{n}\right\|_{\infty} \leq\left\|\omega^{n-1}\right\|_{\infty}+\sigma \Delta t\left\|\omega^{n-1}\right\|_{\infty}+\sigma \Delta t N^{-1}\left\|\boldsymbol{F}^{n}\right\|_{\infty}, \quad n=1,2, \ldots, M .
\end{aligned}
$$


Summing (40) from 1 to $n$, we attain

$$
\left\|\omega^{n}\right\|_{\infty} \leq\left\|\omega^{0}\right\|_{\infty}+\sigma \Delta t \sum_{i=0}^{n-1}\left\|\omega^{i}\right\|_{\infty}+\sigma \Delta t N^{-1} \sum_{i=1}^{n}\left\|\boldsymbol{F}^{i}\right\|_{\infty}, \quad n=1,2, \ldots, M
$$

By the discrete Gronwall inequality (Lemma 4) and from (41) we obtain

$$
\left\|\boldsymbol{\omega}^{n}\right\|_{\infty} \leq\left(\left\|\boldsymbol{\omega}^{0}\right\|_{\infty}+\sigma \Delta t N^{-1} \sum_{i=1}^{n}\left\|\boldsymbol{F}^{i}\right\|_{\infty}\right) \exp [\sigma n \Delta t], \quad n=1,2, \ldots, M
$$

Combining (42) with (39), we get

$$
\left\|\boldsymbol{\psi}^{n}\right\|_{\infty} \leq \sigma N^{-1}\left(\left\|\omega^{0}\right\|_{\infty}+\Delta t N^{-1} \sum_{i=1}^{n}\left\|\boldsymbol{F}^{i}\right\|_{\infty}\right), \quad n=1,2, \ldots, M
$$

Because $\omega_{N}^{n}=\tilde{\boldsymbol{N}} \cdot \boldsymbol{\omega}^{n}, \psi_{N}^{n}=\tilde{\boldsymbol{N}} \cdot \psi^{n}$, and $\|\tilde{\boldsymbol{N}}\|_{\infty} \leq 1$, from (42) and (43) we immediately attain (33) and (34), respectively.

Finally, we discuss the convergence of the CNFSE solutions. Subtracting (29) and (30) from (9) and (10) taking $w=w_{N}$, respectively, we attain the following equations for determining the error:

$$
\begin{aligned}
& \int_{\Theta} \nabla\left(\psi^{n-1}-\psi_{N}^{n-1}\right) \nabla w_{N} \mathrm{~d} x \mathrm{~d} y \\
& =\int_{\Theta}\left(\omega^{n-1}-\omega_{N}^{n-1}\right) w_{N} \mathrm{~d} x \mathrm{~d} y, \quad \forall w_{N} \in V_{N}, n=1,2, \ldots, M+1 ; \\
& \int_{\Theta}\left[\left(\omega^{n}-\omega_{N}^{n}\right) w_{N}+\frac{\mu \Delta t}{2} \nabla\left(\omega^{n}-\omega_{N}^{n}\right) \nabla w_{N}\right] \mathrm{d} x \mathrm{~d} y \\
& =\int_{\Theta}\left(\omega^{n-1}-\omega_{N}^{n-1}\right) w_{N} \mathrm{~d} x \mathrm{~d} y \\
& \quad-\frac{\mu \Delta t}{2} \int_{\Theta} \nabla\left(\omega^{n-1}-\omega_{N}^{n-1}\right) \nabla w_{N} \mathrm{~d} x \mathrm{~d} y, \quad \forall w_{N} \in V_{N}, n=1,2, \ldots, M,
\end{aligned}
$$

where $\omega_{N}^{0}=R_{N} \omega^{0}$.

By (44) and (28), the Cauchy-Schwarz, Hölder, and Poincaré inequalities, and Theorem 7 , we obtain

$$
\begin{aligned}
\| & \nabla\left(\psi^{n-1}-\psi_{N}^{n-1}\right) \|_{0}^{2} \\
= & \int_{\Theta} \nabla\left(\psi^{n-1}-\psi_{N}^{n-1}\right) \nabla\left(\psi^{n-1}-\psi_{N}^{n-1}\right) \mathrm{d} x \mathrm{~d} y \\
= & \int_{\Theta} \nabla\left(\psi^{n-1}-R_{N} \psi^{n-1}\right) \nabla\left(\psi^{n-1}-R_{N} \psi^{n-1}\right) \mathrm{d} x \mathrm{~d} y \\
& \quad+\int_{\Theta} \nabla\left(\psi^{n-1}-\psi_{N}^{n-1}\right) \nabla\left(R_{N} \psi^{n-1}-\psi_{N}^{n-1}\right) \mathrm{d} x \mathrm{~d} y \\
= & \left\|\nabla\left(\psi^{n-1}-R_{N} \psi^{n-1}\right)\right\|_{0}^{2}+\int_{\Theta}\left(\omega^{n-1}-\omega_{N}^{n-1}\right)\left(R_{N} \psi^{n-1}-\psi_{N}^{n-1}\right) \mathrm{d} x \mathrm{~d} y \\
\leq & \left\|\nabla\left(\psi^{n-1}-R_{N} \psi^{n-1}\right)\right\|_{0}^{2}+\left\|\omega^{n-1}-\omega_{N}^{n-1}\right\|_{0}\left\|R_{N} \psi^{n-1}-\psi^{n-1}\right\|_{0}
\end{aligned}
$$




$$
\begin{aligned}
& +\left\|\omega^{n-1}-\omega_{N}^{n-1}\right\|_{0}\left\|\psi^{n-1}-\psi_{N}^{n-1}\right\|_{0} \\
\leq & \sigma\left(N^{2-2 q}+\left\|\omega^{n-1}-\omega_{N}^{n-1}\right\|_{0}^{2}\right) \\
& +\frac{1}{2}\left\|\nabla\left(\psi^{n-1}-\psi_{N}^{n-1}\right)\right\|_{0}^{2}, \quad n=1,2, \ldots, M+1,2 \leq q \leq N+1 .
\end{aligned}
$$

Further, we get

$$
\begin{aligned}
& \left\|\nabla\left(\psi^{n-1}-\psi_{N}^{n-1}\right)\right\|_{0} \\
& \quad \leq \sigma\left(N^{1-q}+\left\|\omega^{n-1}-\omega_{N}^{n-1}\right\|_{0}\right), \quad n=1,2, \ldots, M+1,2 \leq q \leq N+1 .
\end{aligned}
$$

By using (45) and (28), the Hölder, Poincaré, and Cauchy-Schwarz inequalities, and Theorem 7 , we obtain

$$
\begin{aligned}
&\left\|\omega^{n}-\omega_{N}^{n}\right\|_{0}^{2}+\frac{\mu \Delta t}{2}\left\|\nabla\left(\omega^{n}-\omega_{N}^{n}\right)\right\|_{0}^{2} \\
&=\int_{\Theta}\left(\omega^{n}-\omega_{N}^{n}\right)\left(\omega^{n}-\omega_{N}^{n}\right) \mathrm{d} x \mathrm{~d} y+\frac{\mu \Delta t}{2} \int_{\Theta} \nabla\left(\omega^{n}-\omega_{N}^{n}\right) \nabla\left(\omega^{n}-\omega_{N}^{n}\right) \mathrm{d} x \mathrm{~d} y \\
&=\int_{\Theta}\left(\omega^{n}-\omega_{N}^{n}\right)\left(\omega^{n}-R_{N} \omega^{n}\right) \mathrm{d} x \mathrm{~d} y+\frac{\mu \Delta t}{2}\left\|\nabla\left(\omega^{n}-R_{N} \omega^{n}\right)\right\|_{0}^{2} \\
& \quad+\int_{\Theta}\left(\omega^{n}-\omega_{N}^{n}\right)\left(R_{N} \omega^{n}-\omega_{N}^{n}\right) \mathrm{d} x \mathrm{~d} y \\
& \quad+\frac{\mu \Delta t}{2} \int_{\Theta} \nabla\left(\omega^{n}-\omega_{N}^{n}\right) \nabla\left(R_{N} \omega^{n}-\omega_{N}^{n}\right) \mathrm{d} x \mathrm{~d} y \\
&=\int_{\Theta}\left(\omega^{n}-\omega_{N}^{n}\right)\left(\omega^{n}-R_{N} \omega^{n}\right) \mathrm{d} x \mathrm{~d} y+\frac{\mu \Delta t}{2}\left\|\nabla\left(\omega^{n}-R_{N} \omega^{n}\right)\right\|_{0}^{2} \\
& \quad+\int_{\Theta}\left(\omega^{n-1}-\omega_{N}^{n-1}\right)\left(R_{N} \omega^{n}-\omega^{n}\right) \mathrm{d} x \mathrm{~d} y+\int_{\Theta}\left(\omega^{n-1}-\omega_{N}^{n-1}\right)\left(\omega^{n}-\omega_{N}^{n}\right) \mathrm{d} x \mathrm{~d} y \\
& \quad-\frac{\mu \Delta t}{2} \int_{\Theta} \nabla\left(\omega^{n-1}-R_{N} \omega^{n-1}\right) \nabla\left(R_{N} \omega^{n}-\omega^{n}\right) \mathrm{d} x \mathrm{~d} y \\
& \quad-\frac{\mu \Delta t}{2} \int_{\Theta} \nabla\left(\omega^{n-1}-\omega_{N}^{n-1}\right) \nabla\left(\omega^{n}-\omega_{N}^{n}\right) \mathrm{d} x \mathrm{~d} y \\
& \leq \sigma N^{-q}\left(\left\|\omega^{n}-\omega_{N}^{n}\right\|_{0}+\left\|\omega^{n-1}-\omega_{N}^{n-1}\right\|_{0}\right)+\sigma \Delta t N^{2-2 q} \\
&+\frac{\mu \Delta t}{4}\left\|\nabla\left(\omega^{n-1}-R_{N} \omega^{n-1}\right)\right\|_{0}^{2}+\frac{\mu \Delta t}{4}\left\|\nabla\left(\omega^{n}-R_{N} \omega^{n}\right)\right\|_{0}^{2} \\
&+\frac{1}{2}\left\|\omega^{n-1}-\omega_{N}^{n-1}\right\|_{0}^{2}+\frac{1}{2}\left\|\omega^{n}-\omega_{N}^{n}\right\|_{0}^{2}, \quad n=1,2, \ldots, M, 2 \leq q \leq N+1 .
\end{aligned}
$$

Further, we get

$$
\begin{aligned}
\left\|\omega^{n}-\omega_{N}^{n}\right\|_{0}^{2}+\frac{\mu \Delta t}{2}\left\|\nabla\left(\omega^{n}-\omega_{N}^{n}\right)\right\|_{0}^{2} \\
\leq\left\|\omega^{n-1}-\omega_{N}^{n-1}\right\|_{0}^{2}+\frac{\mu \Delta t}{2}\left\|\nabla\left(\omega^{n-1}-\omega_{N}^{n-1}\right)\right\|_{0}^{2} \\
\quad+\sigma N^{-q}\left(\left\|\omega^{n}-\omega_{N}^{n}\right\|_{0}+\left\|\omega^{n-1}-\omega_{N}^{n-1}\right\|_{0}\right)+\sigma \Delta t N^{2-2 q}, \quad n=1,2, \ldots, M .
\end{aligned}
$$


Summing (49) from 1 to $n$ and using Theorem 7, we attain

$$
\begin{aligned}
& \left\|\omega^{n}-\omega_{N}^{n}\right\|_{0}^{2}+\frac{\mu \Delta t}{2}\left\|\nabla\left(\omega^{n}-\omega_{N}^{n}\right)\right\|_{0}^{2} \\
& \leq\left\|\omega^{0}-R_{N} \omega^{0}\right\|_{0}^{2}+\frac{\mu \Delta t}{2}\left\|\nabla\left(\omega^{0}-R_{N} \omega^{0}\right)\right\|_{0}^{2} \\
& \quad+\sigma N^{-1} \sum_{i=0}^{n}\left\|\omega^{i}-\omega_{N}^{i}\right\|_{0}^{2}+\sigma\left(N^{2-2 q}+n \Delta t N^{2-2 q}\right) \\
& \leq \sigma\left(N^{2-2 q}+n \Delta t N^{2-2 q}\right)+\frac{1}{2 N} \sum_{i=0}^{n}\left\|\omega^{i}-\omega_{N}^{i}\right\|_{0}^{2}, \quad n=1,2, \ldots, M .
\end{aligned}
$$

When $N$ is sufficiently large such that $N^{-1} \leq 1 / 2$, from (50) we attain

$$
\begin{aligned}
& \left\|\omega^{n}-\omega_{N}^{n}\right\|_{0}^{2}+\Delta t\left\|\nabla\left(\omega^{n}-\omega_{N}^{n}\right)\right\|_{0}^{2} \\
& \quad \leq \sigma N^{2-2 q}+N^{-1} \sum_{i=0}^{n-1}\left\|\omega^{i}-\omega_{N}^{i}\right\|_{0}^{2}, \quad n=1,2, \ldots, M .
\end{aligned}
$$

By the discrete Gronwall inequality (Lemma 4) and from (51) we obtain

$$
\begin{aligned}
& \left\|\omega^{n}-\omega_{N}^{n}\right\|_{0}^{2}+\Delta t\left\|\nabla\left(\omega^{n}-\omega_{N}^{n}\right)\right\|_{0}^{2} \\
& \quad \leq \sigma N^{2-2 q} \exp \left(n N^{-1}\right) \\
& \quad \leq \sigma N^{1-2 q}, \quad n=1,2, \ldots, M, 2 \leq q \leq N+1 .
\end{aligned}
$$

By (52) and Theorem 5 we obtain (35). Combining (52) with (47) and Theorem 5, we attain (36). This finishes the proof of Theorem 10.

Because $\omega=\partial v / \partial x-\partial u / \partial y$ and $\omega_{N}^{n}=\partial v_{N}^{n} / \partial x-\partial u_{N}^{n} / \partial y$, we immediately attain the following result.

Theorem 11 Under the same conditions as Theorems 5 and 10, the 2D non-stationary Stokes equations about vorticity-stream functions, Problem 1, has a unique set of fluid velocity CNFSE solutions $\left(u_{N}^{n}, v_{N}^{n}\right)$ holding the following stability:

$$
\left\|u_{N}^{n}\right\|_{0, \infty}+\left\|v_{N}^{n}\right\|_{0, \infty} \leq \sigma\left[\left\|u^{0}\right\|_{0, \infty}+\left\|v^{0}\right\|_{0, \infty}+\Delta t N^{-1} \sum_{i=1}^{n}\left(\left\|g_{1}^{i}\right\|_{1, \infty}+\left\|g_{2}^{i}\right\|_{1, \infty}\right)\right]
$$

and the following convergence:

$$
\left\|\partial_{y}\left(u\left(x, y, t_{n}\right)-u_{N}^{n}\right)\right\|_{0}+\left\|\partial_{x}\left(v\left(x, y, t_{n}\right)-v_{N}^{n}\right)\right\|_{0} \leq \sigma\left(\Delta t^{2}+N^{1-q}\right),
$$

where $n=1,2, \ldots, M$ and $2 \leq q \leq N+1$.

Remark 12 The error estimates in Theorem 11 attain optimal order even if $\Theta$ is the polygonal bounded domain and there is only $(u, v) \in H^{2}\left(0, T ; H_{0}^{1}(\Theta) \cap H^{2}(\Theta)\right)$. Especially, the system of equations (37) has sparse block-diagonal matrices with $4 \times 4$-blocks such that 
we enable to solve these equations numerically up to very large size of matrices by means of the chasing algorithm working with this kind of matrices by MATLAB software (see $[10,29])$.

\section{Two numerical examples}

In this section, we utilize three sets of numerical examples to verify the correctness of the theoretical results of the CNFSE format, i.e., Problem 8, for the 2D non-stationary Stokes equations about vorticity-stream functions. These numerical simulations are implemented by Matlab software on Microsoft Surface Book-Computer with Int Core i7 Processor and 16 GB RAM.

\subsection{The numerical example of square cavity flow}

In this numerical example, we choose the computational field $\Theta=(0,1) \times(0,1), \operatorname{Re}=10^{3}$, the side length $\Delta x=\Delta y=0.01$ of quadrilateral elements in $\Im_{N}$, i.e., $N=10^{4}$, the time step $\Delta t=0.0001$, the source vector function $\left(g_{1}(x, y, t), g_{2}(x, y, t)\right)=(0,0)$, the initial velocity vector $\left(u^{0}(x, y), v^{0}(x, y)\right)=(1,0)$ on $0 \leq x \leq 1$ and $y=1$ but $\left(u^{0}(x, y), v^{0}(x, y)\right)=(0,0)$ on other part of $\bar{\Theta}$, the boundary value velocity vector $\left(\varphi_{u}(x, y, t), \varphi_{v}(x, y, t)\right)=(1,0)$ on $0 \leq x \leq 1$ and $y=1$ at $t=0$ but $\left(\varphi_{u}(x, y, t), \varphi_{v}(x, y, t)\right)=(0,0)$ on other sides of $\partial \Theta$ and at other moments. Thus, we can conclude from $\left\|\partial_{y}\left(u_{N}^{n-1}-u_{N}^{n}\right)\right\|_{0}+\left\|\partial_{x}\left(v_{N}^{n-1}-v_{N}^{n}\right)\right\|_{0}=O\left(\Delta t^{2}, N^{-2}\right)$ that the theoretical errors for the CNFSE solutions are $O\left(10^{-8}\right)$.

By the CNFSE model, i.e., Problem 2, we can compute out the CNFSE solution at $t=3$, depicted in Fig. 1. And the absolute error when $t=3$, estimated by $\left\|\partial_{y}\left(u_{N}^{n-1}-u_{N}^{n}\right)\right\|_{0}+$ $\left\|\partial_{x}\left(v_{N}^{n-1}-v_{N}^{n}\right)\right\|_{0}(1 \leq n \leq 30,000)$, is depicted in Fig. 2, which are accorded with the theoretical conclusion, because both errors are no more than $O\left(10^{-8}\right)$. This implies that the CNFSE model is efficient and feasible for solving the 2D non-stationary Stokes equations about vorticity-stream functions.

\subsection{The numerical example of channel flow with two identical rectangular protrusions}

The computational domain $\Theta$ consists of a channel with a width of 6 and a total length of 20 , with two identical rectangular protrusions at the bottom and at the top of the channel. The two rectangular protrusions both have a width of 2 and a length of 4 (see Fig. 3).

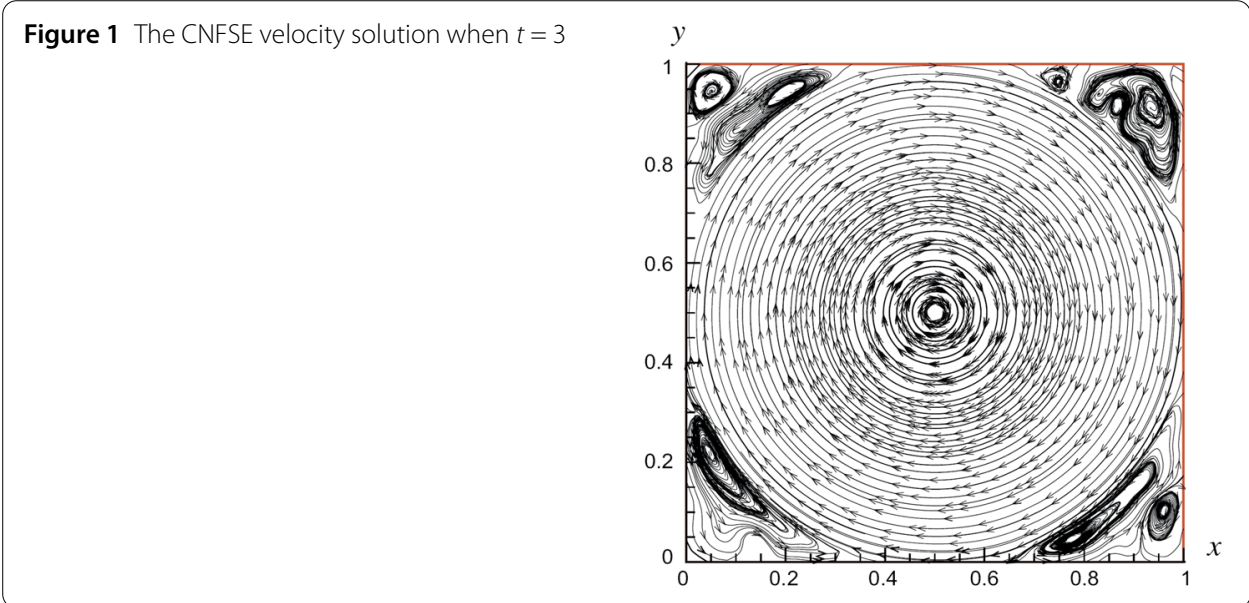




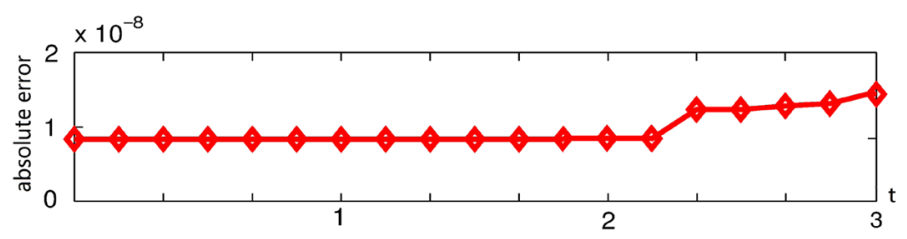

Figure 2 The absolute error when $0 \leq t \leq 3$ estimated by $\left\|\partial_{y}\left(u_{N}^{n-1}-u_{N}^{n}\right)\right\|_{0}+\left\|\partial_{x}\left(v_{N}^{n-1}-v_{N}^{n}\right)\right\|_{0}(1 \leq n \leq 30,000)$
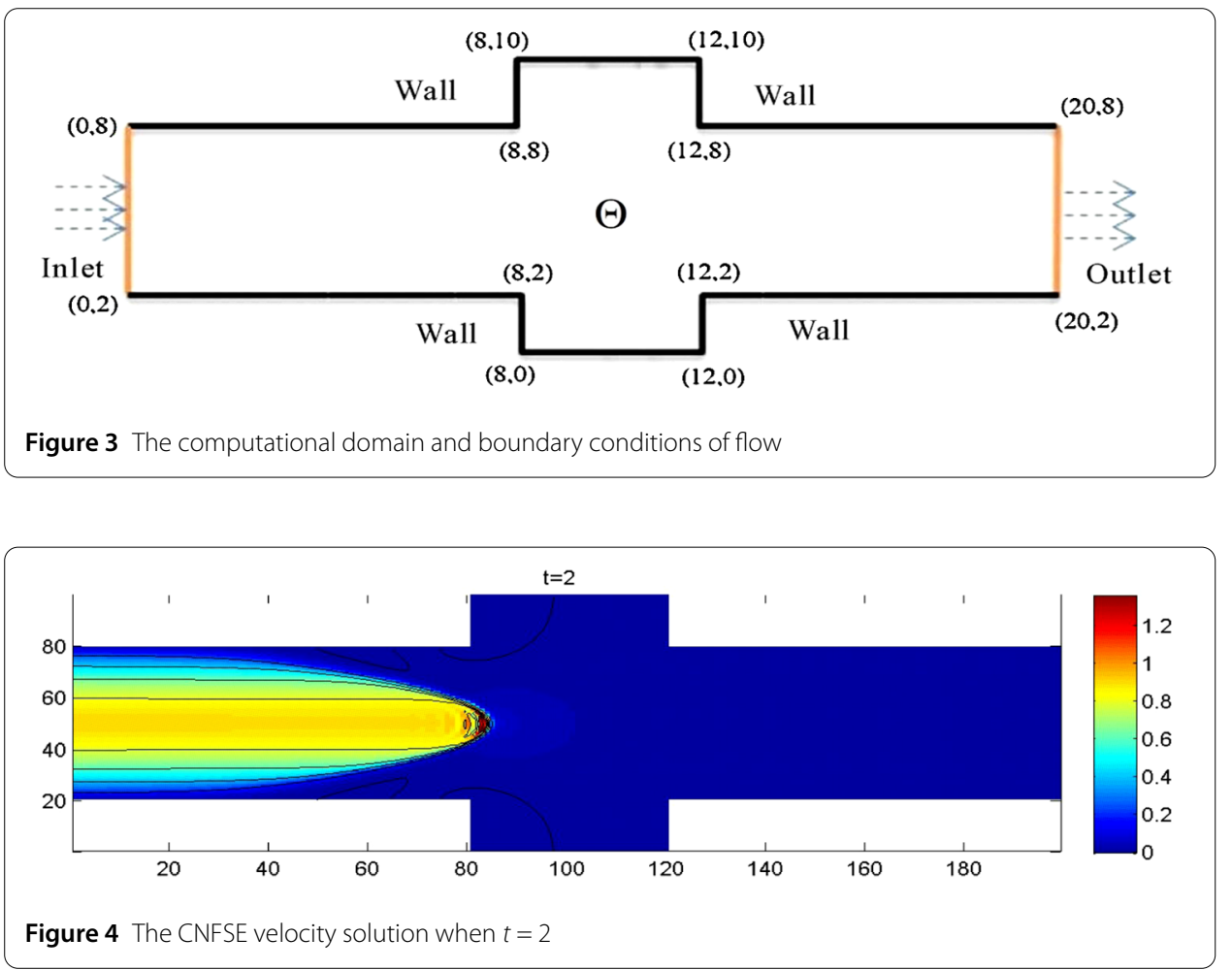

A structured mesh with side length $\Delta x=\Delta y=0.01$ is used. Except for the inflow from the left boundary with a velocity of $(u, v)=(0.1(y-2)(8-y), 0)(x=0,2 \leq y \leq 8)$ and the outflow on the right boundary with velocity of $u(x, y, t)=u(20-1 / N, y, t)(20-1 / N \leq x \leq 20$, $2 \leq y \leq 8,0 \leq t \leq T)$, all of the initial and other boundary value conditions are taken as 0 . The time-step increment is also taken as $\Delta t=0.0001$. In this case, the theoretical errors also attain $O\left(10^{-8}\right)$.

By the CNFSE model, i.e., Problem 2, we can compute out the CNFSE solutions at $t=$ 2, 3, 4, depicted in Figs. 4, 5, and 6, respectively. And the absolute error when $0 \leq t \leq 4$, estimated by $\left\|\partial_{y}\left(u_{N}^{n-1}-u_{N}^{n}\right)\right\|_{0}+\left\|\partial_{x}\left(v_{N}^{n-1}-v_{N}^{n}\right)\right\|_{0}(1 \leq n \leq 40,000)$, is depicted in Fig. 7, which are accorded with the theoretical conclusions, because both errors are no more than $O\left(10^{-8}\right)$. This implies that the CNFSE model is valid and feasible for solving the $2 \mathrm{D}$ non-stationary Stokes equations about vorticity-stream functions.

\subsection{The numerical example with analytical solution}

In this numerical example, we choose the computational field $\Theta=(0, \pi) \times(0, \pi)$, the source function $f(x, y, t)=(1+2 \mu) \exp (t) \sin x \sin y$, and $\omega^{0}=\sin x \sin y$ in (4). Thus, 


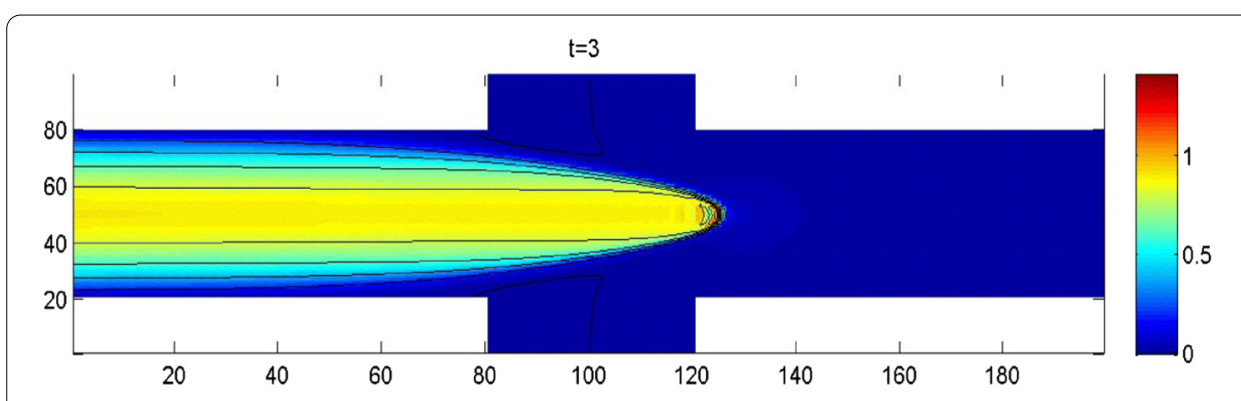

Figure 5 The CNFSE velocity solution when $t=3$
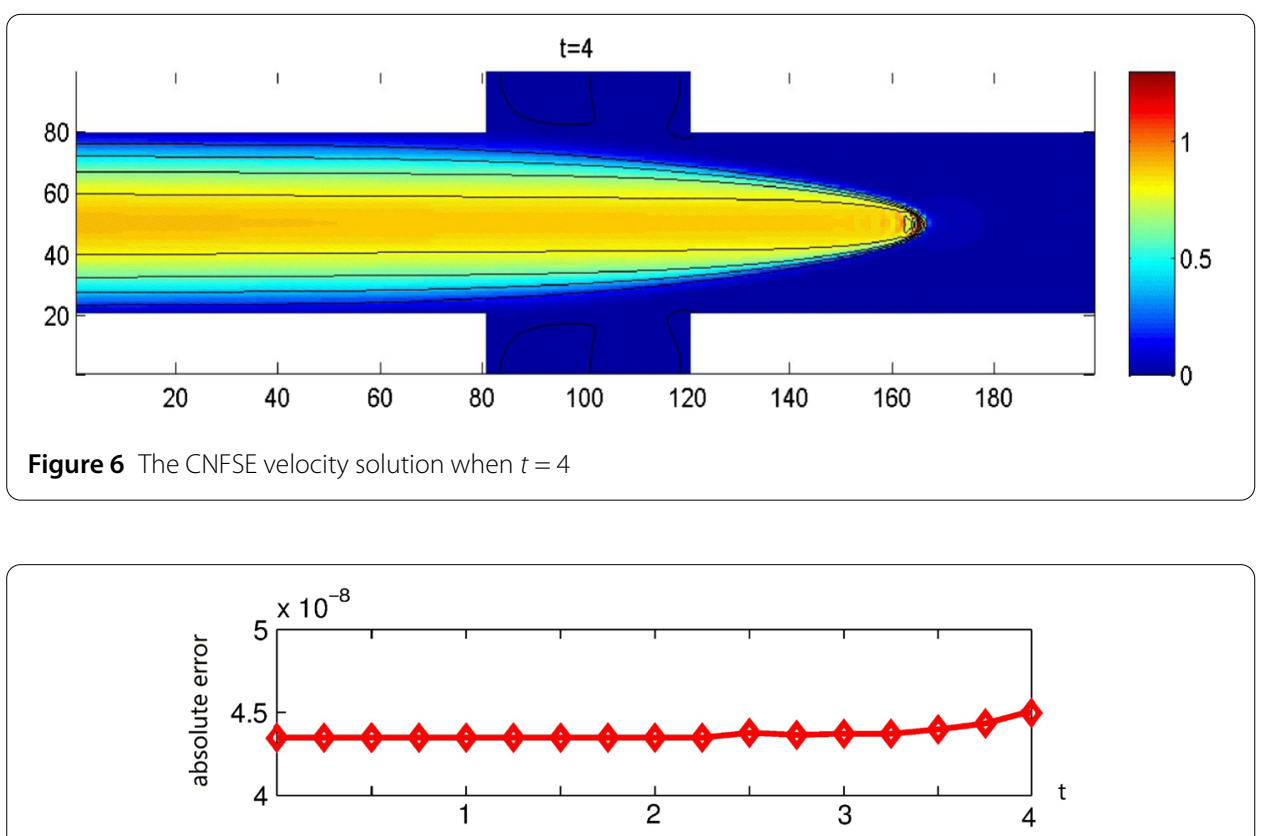

Figure 7 The absolute error when $0 \leq t \leq 4$ estimated by $\left\|\partial_{y}\left(u_{N}^{n-1}-u_{N}^{n}\right)\right\|_{0}+\left\|\partial_{x}\left(v_{N}^{n-1}-v_{N}^{n}\right)\right\|_{0}(1 \leq n \leq 40,000)$

Table 1 The errors between the numerical solutions and the analytical solution at $t=1$

\begin{tabular}{lll}
\hline$\Delta t$ and $N$ & $\left\|\nabla\left(\psi\left(x, y, t_{n}\right)-\psi_{N}^{n}\right)\right\|_{0}$ & $\left\|\omega\left(x, y, t_{n}\right)-\omega_{N}^{n}\right\|_{0}$ \\
\hline$\Delta t=1 / 8$ and $N=8$ & $2.0951 \mathrm{e}-2$ & $1.5036 \mathrm{e}-2$ \\
$\Delta t=1 / 16$ and $N=16$ & $4.7351 \mathrm{e}-3$ & $3.5732 \mathrm{e}-3$ \\
$\Delta t=1 / 32$ and $N=32$ & $5.7826 \mathrm{e}-4$ & $4.9875 \mathrm{e}-4$ \\
$\Delta t=1 / 64$ and $N=64$ & $2.3564 \mathrm{e}-4$ & $1.3161 \mathrm{e}-4$ \\
\hline
\end{tabular}

Eqs. (3) and (4) have a set of analytical solutions: $\psi=\omega / 2=\frac{1}{2} \exp (t) \sin x \sin y$ and $\omega=$ $\exp (t) \sin x \sin y$.

When $R e=10^{3}$, i.e., $\mu=10^{-3}$, we estimate the errors between the numerical solutions and the analytical solutions with different time steps and numbers of meshes at $t=1$ and 2 , shown in Tables 1 and 2, respectively.

Tables 1 and 2 show that the numerically computing errors are accorded with the theoretical results in Theorem 10, i.e., both errors are second-order accuracy since $(1 / 8)^{2}=$ $O\left(10^{-2}\right),(1 / 16)^{2}=O\left(10^{-3}\right),(1 / 32)^{2}=O\left(10^{-4}\right)$, and $(1 / 64)^{2}=O\left(10^{-4}\right)$. Due to the accumulation of round-off error, the numerically computing errors at $t=2$ are larger than those at 
Table 2 The errors between the numerical solutions and the analytical solution at $t=2$

\begin{tabular}{lll}
\hline$\Delta t$ and $N$ & $\left\|\nabla\left(\psi\left(x, y, t_{n}\right)-\psi_{N}^{n}\right)\right\|_{0}$ & $\left\|\omega\left(x, y, t_{n}\right)-\omega_{N}^{n}\right\|_{0}$ \\
\hline$\Delta t=1 / 8$ and $N=8$ & $4.4732 \mathrm{e}-2$ & $3.3764 \mathrm{e}-2$ \\
$\Delta t=1 / 16$ and $N=16$ & $7.0124 \mathrm{e}-3$ & $6.8274 \mathrm{e}-3$ \\
$\Delta t=1 / 32$ and $N=32$ & $9.5675 \mathrm{e}-4$ & $8.9906 \mathrm{e}-4$ \\
$\Delta t=1 / 64$ and $N=64$ & $4.6703 \mathrm{e}-4$ & $3.1033 \mathrm{e}-4$ \\
\hline
\end{tabular}

$t=1$, which is reasonable. This further shows that the CNFSE model is efficient and feasible for finding the numerical solutions of the 2D non-stationary Stokes equations about vorticity-stream functions.

\section{Conclusions and discussion}

In this work, we have established the time semi-discretized CN and CNFSE format for the 2D non-stationary Stokes equations about vorticity-stream functions and analyzed the existence, uniqueness, stability, and convergence of the time semi-discretized $\mathrm{CN}$ and CNFSE solutions, respectively. We have also used three sets of numerical examples to check the feasibility and effectiveness of the CNFSE format and to verity that the numerical computing consequences are accorded with the theoretical analysis ones. Moreover, it is shown that the CNFSE format is valid for solving the 2D non-stationary Stokes equations about vorticity-stream functions.

Although we here only research the CNFSE method for the 2D non-stationary Stokes equations about vorticity-stream functions, the CNFSE method can easily and effectively be used to solve for the non-stationary Stokes equations in three-dimensional space or more complex fluid dynamics equations, even be applied in the more complex real-world engineering problems. Therefore, our technique is promising as regards applications.

\section{Acknowledgements}

The authors are thankful to the honorable Reviewers and Editors for their valuable suggestions and comments, which improved the paper.

\section{Funding}

This research was supported by the National Key Research and Development Project of China Grant 2017YFC1500301, the National Science Foundation of China grants 11671106 and 41704047, Qian Science Cooperation Platform Talent Grant [2017]5726-41, and the "Double Tops" Postgraduate Talent Cultivation Project of North China Electric Power University Grant XM1805313.

\section{Availability of data and materials}

The authors declare that all data and material in the paper are available and veritable.

Competing interests

The authors declare that they have no competing interests.

Authors' contributions

All authors contributed equally and significantly in writing this article. All authors wrote, read and approved the final manuscript.

\section{Author details}

${ }^{1}$ School of Science, Beijing Technology and Business University, Beijing, China. ${ }^{2}$ School of Mathematics and Physics, North China Electric Power University, Beijing, China.

\section{Publisher's Note}

Springer Nature remains neutral with regard to jurisdictional claims in published maps and institutional affiliations. 


\section{References}

1. Girault, V., Raviart, P.A.: Finite Element Methods for Navier-Stokes Equations: Theory and Algorithms. Springer, Berlin (1986)

2. Temam, R.: Navier-Stokes Equations, 3rd edn. North-Holland, Amsterdam (1984)

3. Heywood, J.G., Rannacher, R.: Finite element approximation of the non-stationary Navier-Stokes problem, I. Regularity of solutions and second order estimates for spatial discretization. SIAM J. Numer. Anal. 19(2), 275-311 (1982)

4. Verfürth, R:: A posteriori error analysis of space-time finite element discretizations of the time-dependent Stokes equations. Calcolo 47(3), 149-167 (2010)

5. Isaev, V.I., Shapeev, V.P.: High-accuracy versions of the collocations and least squares method for the numerical solution of the Navier-Stokes equations. Comput. Math. Math. Phys. 50(10), 1670-1681 (2010)

6. Botella, O., Peyret, R.: Benchmark spectral results on the lid-driven cavity flow. Comput. Fluids 27(4), 421-433 (1998)

7. Barragy, E., Carey, G.F.: Stream function-vorticity driven cavity solution using $p$ finite elements. Comput. Fluids 26(5), 453-468 (1997)

8. Erturk, E., Gokcol, C.: Fourth order compact formulation of Navier-Stokes equations and driven cavity flow at high Reynolds numbers. Int. J. Numer. Methods Fluids 50(4), 421-436 (2006)

9. Chung, T.J.: Computational Fluid Dynamics. Cambridge University Press, Cambridge (2002)

10. Zhang, W.S.: Finite Difference Methods for Patial Differential Equations in Science Computation. Higher Education Press, Beijing (2006)

11. Luo, Z.D.: The Foundations and Applications of Mixed Finite Element Methods. Chinese Science Press, Beijing (2006) (in Chinese)

12. Heywood, J.G., Rannacher, R.: Finite element approximation of the non-stationary Navier-Stokes problem, IV: error analysis for second-order time discretization. SIAM J. Numer. Anal. 27(2), 353-384 (1990)

13. Cai, Z., McCormick, S.: On the accuracy of the finite volume element method for diffusion equations on composite grid. SIAM J. Numer. Anal. 27(3), 636-655 (1990)

14. Li, J., Chen, Z.X.: A new stabilized finite volume method for the stationary Stokes equations. Adv. Comput. Math. 30(2), 141-152 (2009)

15. Baltensperger, R., Trummer, M.R.: Spectral differencing with a twist. SIAM J. Sci. Comput. 24(5), 1465-1487 (2002)

16. Canuto, C., Hussaini, M.Y., Quarteroni, A., Zang, T.A.: Spectral Methods in Fluid Dynamics. Springer, Berlin (2012)

17. Guo, B.Y.: Spectral Methods and Their Applications. World Scientific, Singapore (1998)

18. Guo, B.Y.: Some progress in spectral methods. Sci. China Math. 56(12), 2411-2438 (2013)

19. Shen, J., Tang, T.: Spectral and High-Order Methods with Applications. Science Press, Beijing (2006)

20. An, J., Luo, Z.D., Li, H., Sun, P.: Reduced-order extrapolation spectral-finite difference scheme based on POD method and error estimation for three-dimensional parabolic equation. Front. Math. China 10(5), 1025-1040 (2015)

21. Luo, Z.D., Jin, S.J.: A reduced-order extrapolation spectral-finite difference scheme based on the POD method for 2D second-order hyperbolic equations. Math. Model. Anal. 22(5), 569-586 (2017)

22. Guba, O., Taylor, M., St-Cyr, A.: Optimization-based limiters for the spectral element method. J. Comput. Phys. 267(267), 176-195 (2014)

23. Gopalakrishnan, S., Chakraborty, A., Roy Mahapatra, D.: Spectral Finite Element Method. Computational Fluid and Solid Mechanics Series. Springer, Berlin (2007)

24. Karniadakis, G.E., Sherwin, S.J.: Spectral/hp Element Methods for Computational Fluid Dynamic, 2nd edn. Oxford University Press, Oxford (2005)

25. Wang, J.P.: A finite spectral finite element method for incompressible Navier-Stokes equations. Int. J. Mech. Res. 3(3) 33-42 (2014)

26. Hu, Y.Y., Xie, J., Zhang, W.: Solution of two dimensional incompressible Navier-Stokes equation by parallel spectral finite element method. J. Comput. Appl. 37(1), 42-47 (2017)

27. Adams, R.A.: Sobolev Spaces. Academic Press, New York (1975)

28. Luo, Z.D., Chen, G.: Proper Orthogonal Decomposition Methods for Partial Differential Equations. Mathematics in Science and Engineering. Elsevier, Amsterdam (2018). https://www.elsevier.com/books/proper-orthogonaldecomposition-methods-for-partial-differential-equations/luo/978-0-12-816798-4

29. Redfern, D., Campbell, C.: The Matlab ${ }^{\oplus} 5$ Handbook. Springer, New York (1998)

\section{Submit your manuscript to a SpringerOpen ${ }^{\circ}$ journal and benefit from:}

- Convenient online submission

- Rigorous peer review

- Open access: articles freely available online

- High visibility within the field

- Retaining the copyright to your article

Submit your next manuscript at $>$ springeropen.com 